\title{
Bioanalysis
}

\section{Mass spectrometry - an alternative in growth hormone measurement}

Growth hormone $(\mathrm{GH})$ constitutes a set of closely related protein isoforms. In clinical practice, the disagreement of test results between commercially available ligand-binding assays is still an ongoing issue, and incomplete knowledge about the particular function of the different forms leaves an uncertainty of what should be the appropriate measurand. Mass spectrometry is promising to be a way forward. Not only is it capable of providing SI-traceable reference values for the calibration of current GH-tests, but it also offers an independent approach to highly reliable mass-selective quantification of individual GH-isoforms. This capability may add to reliability in doping control too. The article points out why and how.

\section{Measurand}

Growth hormone $(\mathrm{GH})$ is a heterogeneous polypeptide consisting of a number of different isoforms and variants [1]. These are closely related to one another as originating from the same gene cluster (i.e., information about GH-amino acid sequence stored on DNA). In reality, however, just parts of this sequence are read when assembling the $\mathrm{GH}$ while others are skipped (alternative splicing). Typically, therefore, the different forms share much of the parent sequence, but not all.

The sequence of the main $\mathrm{GH}$ form $(22 \mathrm{kDa} \mathrm{GH})$ is shown in Figure 1. This form accounts for $58-82 \%$ of the circulating GH [1]. There is another one, $20 \mathrm{kDa} \mathrm{GH}$, which is lacking in 15 out of the 191 amino acids of the main form. These are marked by grayfilled circles in Figure 1. Next to $20 \mathrm{kDa} G H$, which is estimated to make a $3-15 \%$ fraction of GH forms [1], sequence fragments consisting of amino acids 1-43 have been identified and the complementary stretch (44-191) has been detected in blood [2].

Further complicating matters, different variants may exist of the same isoform by the modification of the side chains, typically resulting from deamidation or glycosylation. Last, but unlikely to complete the list, the hormone has been detected in dimeric or even higher molecular forms [1] (e.g., $[22 \mathrm{kDa}]_{2},[20 \mathrm{kDa}]_{2}$ or $[22-20 \mathrm{kDa}]$ as protein-protein complexes, besides dimers $[22 \mathrm{kDa}]_{2}$ with the monomers linked by disulfide bonds.

Obviously, in this setting, the crucial problem consists in defining which of the abovementioned form(s) is correlating best with the clinical condition(s) they are intended to detect or measure. Finding out about this requires the analytical technology to resolve the different forms while providing reliable quantitative results for each of them.

Mass spectrometry (MS), relying on a fundamentally different principle of analyterecognition enables evaluation of ligandbinding assays (LBAs) without being subject to the same limitations as these. The analytical selectivity generally inherent to MS can be deployed to discriminate isoform-specific $\mathrm{GH}$ sequence fragments. This, in combination with the option to use isotope-labeled internal standards, makes MS an interesting alternative that can contribute to respond to the challenge stated above.

The subsequent discussion is intended to illustrate this fact by the comparison of both kinds of approach, ligand binding versus mass selective.
Cristian G Arsene ${ }^{*, 1}$, Jürgen Kratzsch ${ }^{2}$ \& André Henrion ${ }^{1}$ 'Physikalisch-Technische Bundesanstalt (PTB), Bundesallee 100, 38116 Braunschweig, Germany ${ }^{2}$ Institute for Laboratory Medicine, Clinical Chemistry \& Molecular Diagnostics, University of Leipzig, Paul-List-Straße 13-15, 04103 Leipzig, Germany

*Author for correspondence: christian.arsene@ptb.de 


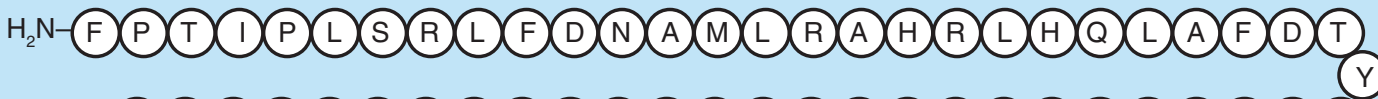

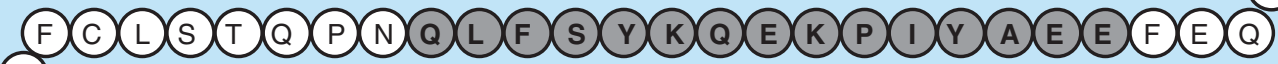

(S)

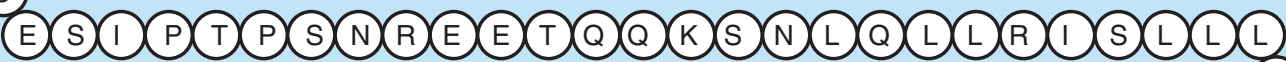

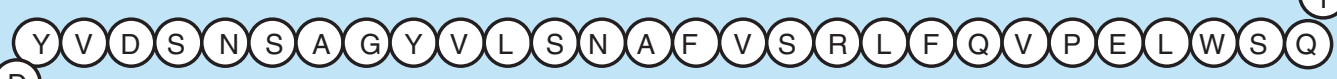

(D)

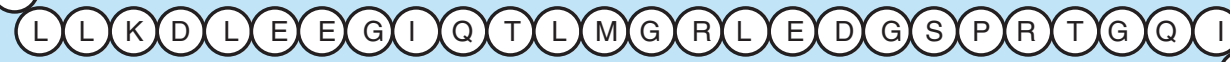

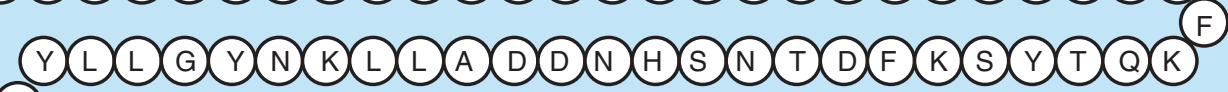

(C)

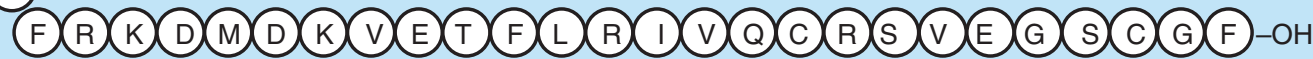

Figure 1. Parent-primary structure (amino acid sequence) of growth hormone. With $22 \mathrm{kDa}$ growth hormone, all 191 amino acids are present, whereas 20 kDa growth hormone lacks in substretch 32-46 (gray-filled circles).

\section{Recognizing by epitope}

\section{Scope \& bounds}

For a long time, the LBAs have been the only way to quantification of GH in biological samples. Apart from this inevitableness, ease of practical implementation and automation, as well as effectiveness in time and costs per analysis are assets of the method principle. Importantly also, sensitivity requirements of clinical tests can be satisfied by use of appropriate detection techniques, as for example, chemiluminescence. Then, detection limits can be achieved of $0.2 \mathrm{ng} / \mathrm{ml}$, or less [3] as, for example, needed for determination of GH-concentrations in patients with GH deficiency.

At the same time, the limited quality of GH-data is still hampering the reliability of conclusions in diagnostic practice. Figure 2 exemplifies this with results returned by testing laboratories in one of the periodic studies organized by RfB DGKL, one of the German providers of External Quality Assessment Services. With both of the serum pools, A and B, the spread of data covers a considerable portion of the range of values typically found in the population as a whole, in spite of the fact, that A and B represent serum of just a single subject, each. Decision limits for diagnostic GH-stimulation tests, for illustration, are in the range of approximately $4-10 \mathrm{ng} / \mathrm{ml}$ [4].

The situation very much resembles what is reported from other countries too. Some further examples describing the impact of the method (laboratory) employed, on patient classification, are provided by Wieringa et al. [5]. In one of them, a factor of 6 , on average, was in-between the results of two different test-kits that had been used with an oral glucose tolerance test [6].

\section{Limiting factors}

A recent review [5] summarizes the most likely causes for intermethod-discrepancies, and why they continue to be unsatisfactory in spite of improvements resulting from standardization/harmonization efforts during these last 10 years.

One category of sources is the different affinity of antibodies toward different forms of GH. In the past, test kits were predominantly based on polyclonal antibodies. These are mixtures of antibodies recognizing different epitopes, each one, on the GH molecule. In this way, in summa, all possible epitopes on the different GH forms are more or less covered. Therefore, polyclonal antibodies may be expected to capture something like 'total-GH' in that sample.

It is different, when monoclonal antibodies are used, as it is the case with most of the more contemporary assays. Uniformly, the same epitope is then recognized. Depending on the presence of this epitope exclusively on just one GH form, or on many forms, the information content will be either of the kind 'total-GH' or 'particular GH form'. Enhanced specificity toward a particular form can be achieved if using two-step immunometric assays with two antibodies recognizing two different epitopes that are only present with that form.

All these antibodies, or antibody-mixtures, capture different fractions out of the spectrum of isoforms present in a given sample, according to the epitope(s) they recognize. Using different methods is equivalent, therefore, to allow for slightly different definitions of the measurand. This obviously accounts for a part of the variation of results if using different methods.

Cross-reactivities of antibodies constitute another potential contribution to intermethod divergences. 
Mistaking a non-GH antigen for $\mathrm{GH}$, as it can happen owing to similarity of epitopes, may result in positive bias. However, it can equally well come to pass inversely. So, for example, if in a sandwich-type assay just one of the two antibodies binds to that antigen, less of GH will be determined than what is present. One example for such antigen is pegvisomant, which may interfere with GH measurement if administered in therapy [7].

However, pegvisomant is present only in sera of treated patients. GH-binding protein (GHBP), which is generally present in serum samples at varying concentration, apparently constitutes still more of a problem. With commercial assays, GH-recovery has been reported to be reduced by up to approximately $40 \%$ as a consequence of GHBP interference [8]. This may be taken as resulting from competition between the binding protein and the antibody for the same GH-epitope.

Apart from these uncertainties, which may be ascribed to variably specific recognition of the analytical target, a more elementary factor contributing to incompatibility has been that, originally, and for a long time, varying GH-preparations have been referred to as primary standards for assay calibration. These were pituitary tissue extracts containing the whole spectrum of isoforms in poorly characterized amount ratios. Additionally aggravating was that measurement was linked to International Units (IU) of biological activity, and not compatible, therefore, with the SI. This, however, has been resolved by a WHO agreement in 2001 [9], about using recombinant $22 \mathrm{kDa} \mathrm{GH}$ (WHO-preparation 98/574) for calibration, from then on, as chemically well-defined pure primary standard. This, at the same time, allows for expression of the results in terms of the (molar) amount of substance, thereby establishing a link to the SI.

Finally, noncommutability of kit-calibrators is discussed as another cause of inaccuracy. 'Commutability' addresses the requirement for the calibrator to behave in the same way as if it was a natural (patient) sample, though most of the time the calibrators are processed serum to which a known amount of GH had been spiked. Noncommutability, is attributed to presence of the analyte in a non-natural form in the calibrator, matrix effects, and lack of specificity of measurement procedures [10], and will obviously affect the applicability of calibration to real samples, and compromise the SI-traceability of results.

\section{Recognizing by mass}

\section{Targeting signature peptides}

Several MS approaches have been developed thus far for measurement of serum $\mathrm{GH}$, on purposes to detect

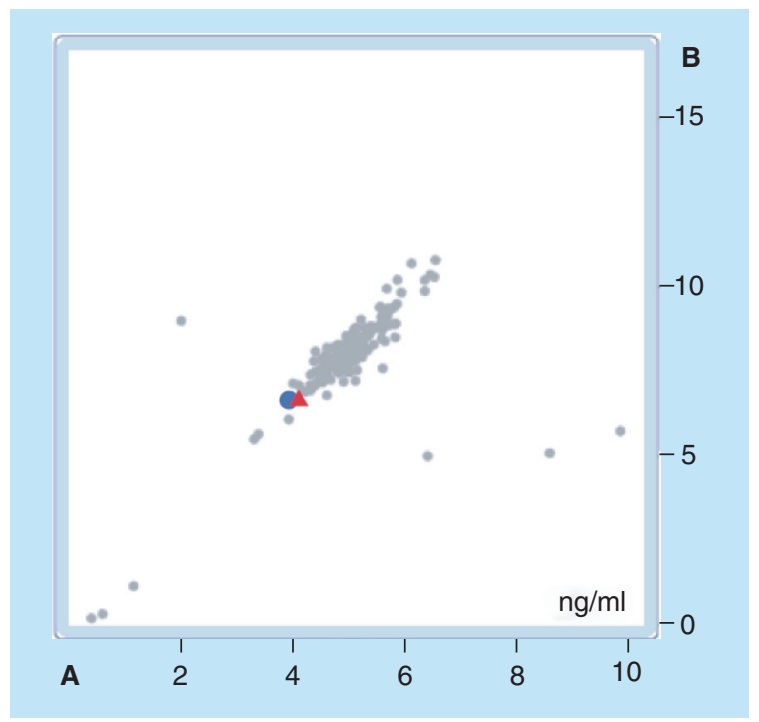

Figure 2. Results in an intercomparison of testing laboratories for the measurand 'growth hormone' (HP4/12) in 2012. A number of 14 different test kits had been employed by 186 participating laboratories. Each laboratory ran two samples (serum pools $\mathbf{A}$ and B) under their routine conditions. Pairs of results (one for pool A, one for B) make one dot in the picture. Red triangle and blue circle: MS results (red: T6-based, blue: T12-based).

Adapted with permission from [44] @ Referenzinstitut für Bioanalytik (2012).

For color images please see www.future-science.com/ doi/full/10.4155/bio.14.196.

illicit administration in stock-breeding and horse-racing $[11,12]$, next to diagnosis of GH-related diseases in human medicine [13-15].

All these methods share in common, that enzymatic cleavage products of $\mathrm{GH}$ are quantified as surrogates (signature peptides) [16], in place of the protein itself. The preference of enzymatic fragments as analytical targets over the intact protein is characteristic for almost exclusively all methods presently being developed in quantitative proteomics. This is obviously owing to the better compatibility with reversed phase chromatography and lower limits of detection/determination attainable with peptides if compared with the larger-sized precursor proteins. It is worth noting that, notwithstanding, the alternative of measuring the intact protein as an option is kept in consideration too [17,18]. This appears mainly motivated by the complementary

\section{Key terms}

SI-traceability: Property of a measurement result whereby the result can be related to a reference through a documented unbroken chain of calibrations.

Signature peptide: Enzymatic cleavage product whose amino acid sequence can be used as unique fingerprint for recognition of the precursor protein (or isoform). 
information, available in this way, about kind and abundance of different molecular variants of the protein.

There are 20 tryptic cleavage points on $22 \mathrm{kDa}$ GH (i.e., after each lysine or arginine) as shown in Figure 3. Of the resulting 21 substretches (products of exhaustive proteolytic cleavage), most can act as an analyte, provided that, they are specific enough in sequence, so as to be excluded from possibly being obtained similarly from any other protein present in the sample. Arsene et al. [13,14,19], for instance, chose T6 and T12 to be quantified. Both sequences had been checked by comparison with the Swiss-Prot data base not to coincide with sequence stretches that might result from any other known human protein.

\section{Integrating isotope-labeled standards}

MS methods developed for clinical GH-measurement almost compellingly have to employ isotope-labeled versions of the analyte as internal standards, so as to achieve the level of quantitative accuracy required. Ideally, the target protein is used to this, since closest mimicking the behavior of the analyte in both, extraction and proteolysis $[20,21]$. In the case of GHmeasurement, recombinantly engineered $22 \mathrm{kDa}$ GH has been employed, either incorporating labeled nitrogen $\left(\mathrm{U}_{-}{ }^{15} \mathrm{~N} \mathrm{GH}\right)[14]$, or labeled amino acids (K and R) [15].

As alternative to whole-protein labeling, isotope labeled peptides (corresponding to the specified enzymatic fragments) have been shown to be applicable as internal standards too [13]. Using labeled peptides as internal standards, of course, takes as a prerequisite that, proteolysis will proceed to completion under the experimental conditions used (see [22] for a discussion).

\section{Extracting targeted peptides}

Once cleavage products have been specified, two fundamentally different strategies for generating and extracting them are conceivable: it is promising to first isolate the protein from the matrix and only then to enzymatically digest the analyte-enriched extract.

This rationale, referred to as 'protein level cleanup', is practiced with the method developments by Bailly-Chouriberry et al. [12] and Le Breton et al. [11], who isolate GH by precipitating it from the serum at its isoelectric point, followed by solid-phase extraction, as well as by Pritchard $e t$ al. [15], who apply twofold solid-phase extraction at different $\mathrm{pH}$ values. Immunoaffinity enrichment is likewise considered as an option for clean-up at the protein level [23,24]. This approach is also referred to as 'MS-based immunoassay', so as characterizing it as a hybrid of two philosophies pursued. However, with respect to the present purpose, employing an antibody-based extraction principle for GH would ostensibly thwart the intent of developing a method that is completely independent of biases associated with analyte selection by epitope recognition.

As opposed to these approaches, the alternative strategy ('peptide level clean-up') appears to be considerably more challenging at first glance, indeed. In this case, the signature peptides have to be recovered after digestion from the serum sample as a whole, which results in

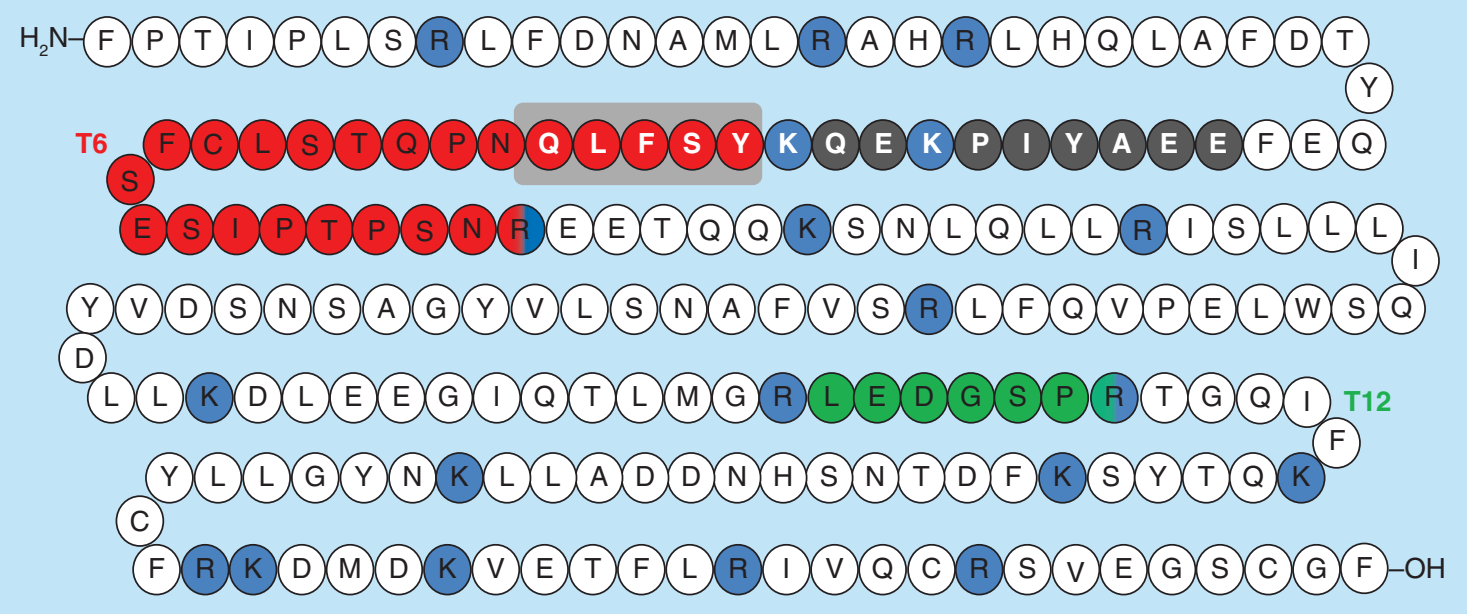

Figure 3. Tryptic cleavage sites on 22 kDa growth hormone. Cleavage occurs after R and K (blue-filled circles). Substretches T6 and T12, used as signature peptides with the method illustrated in Figure 4, are filled with red and green, respectively. T6 is present as shown, only with $22 \mathrm{kDa}$ growth hormone (GH), while the $20 \mathrm{kDa}$ isoform is missing the amino acids within the gray box (cp. Figure 1). T12, on the other hand, will uniformly be produced by both forms. Therefore, T6 captures GH in an isoform-specific way, whereas T12 might be taken as measuring 'total-GH'.

For color images please see www.future-science.com/doi/full/10.4155/bio.14.196. 
a matrix of very high complexity. However, in practice, this has been proven to be manageable both, reliably stable quite as much, as reproducible. An outline of the implementation described by Wagner et al. [19] is given in Figure 4. Data exemplifying accuracy and precision attainable with the type of method are reproduced in Table 1. Remarkably, this appears to be the only MSbased method as yet that has been proven to smoothly be applicable to patient sera within a clinical study [19].

Reversed-phase chromatography followed by strong cation exchange chromatography are used, in this case, for extraction of GH peptides T6 and T12 from the matrix. Similar developments, targeting other proteins, successfully have been using antibodies instead, specifically developed for recognition of the signature peptides [24-26]. This is more than likely to work quite as well with GH-quantification and may even be less time-consuming than chromatographic clean-up.

Using chromatography, on the other hand, obviates the need to develop a separate antibody against every targeted peptide, making it more easy to switch to another peptide, if required with a particular study.

\section{Establishing SI-traceability}

Traceability can be obtained by reference to a calibrator consisting of (GH-stripped) blank serum, which is then fortified with a defined amount of recombinant $22 \mathrm{kDa} \mathrm{GH}$. The calibrator is run according to the same steps and conditions as applied with the sample. The analysis result, eventually, is obtained by relating the signal ratio (peptide/isotope-labeled peptide) measured for the sample, to the ratio obtained for the calibrator. In Figure 4, WHO IS 98/574 is specified as $22 \mathrm{kDa} \mathrm{GH}$ material appropriate for the purpose. However, any other source would do quite as well, as long as it is of the correct amino acid sequence and sufficient in purity.

Value-assignment to the calibrator stock solution is based on amino acid analysis (i.e., complete hydrolysis, quantitatively releasing the individual amino acids). These, in turn, are quantified by isotope dilution MS. In this way, the amount of GH measured for a particular sample is traceable to the (molar) amount of substance of the constituent amino acids, which are simple molecules and readily available as purified reference standards. See [27] for a more detailed discussion of the concept.

\section{GH by MS: scope \& bounds}

\section{Analytical selectivity \& sensitivity}

The promise of MS consists in achieving enhanced selectivity by recognition of analyte mass, rather than epitope, as principle for distinction from the background. Essentially, the data shown in Table 1 account

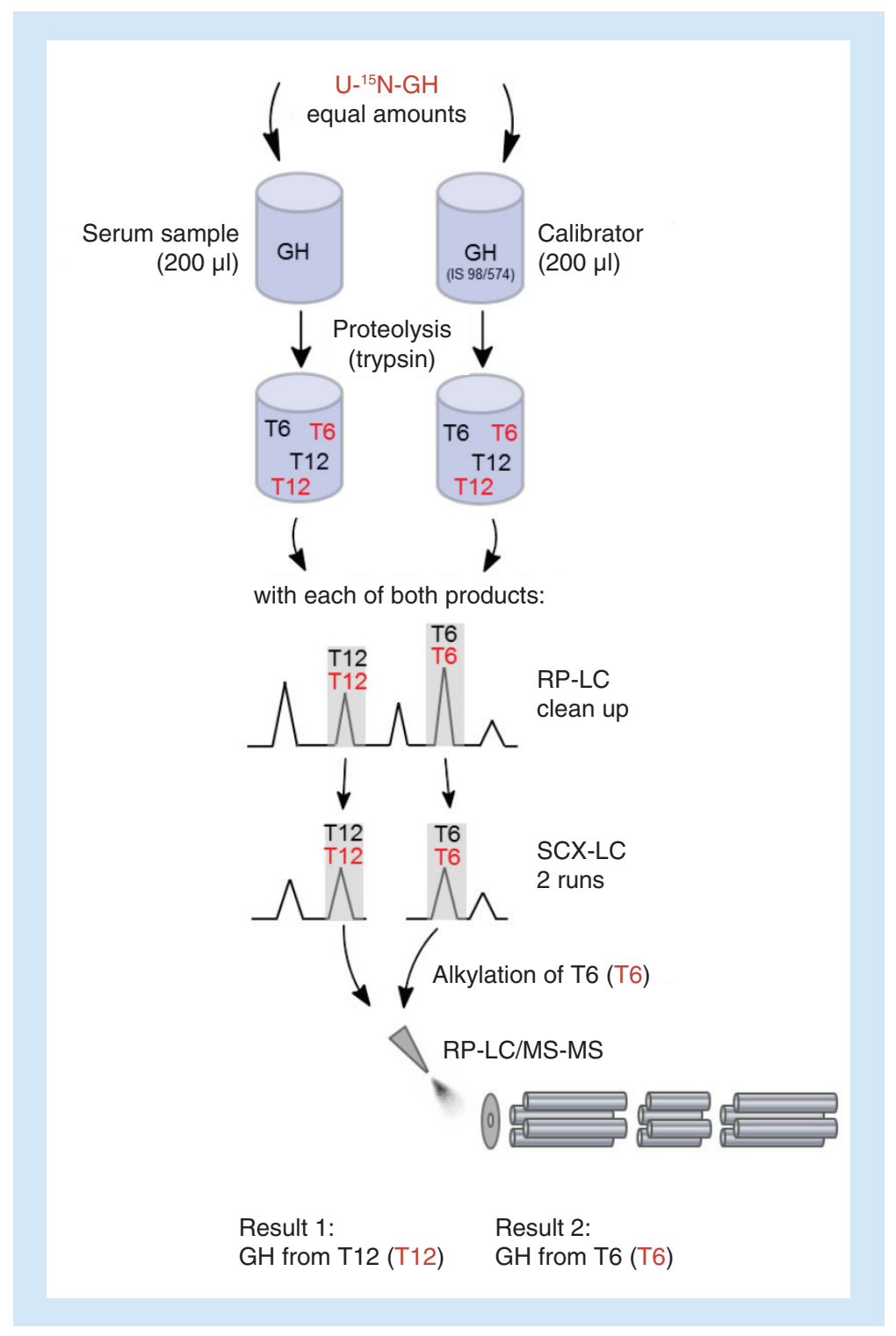

Figure 4. Determination of growth hormone by isotope dilution mass spectrometry using peptide-level clean-up. The serum sample containing $\mathrm{GH}$ is enzymatically cleaved using trypsin as protease. Enzymatic GH fragments, peptides T6 and T12 are quantified as surrogates in place of the intact protein. Along with each individual serum sample, a calibrator (serum plus a known amount of $22 \mathrm{kDa} \mathrm{GH}$, here: WHO IS 98/574) is run and analyzed using the same procedure as with the sample. Isotopically labeled GH (here: $\mathrm{U}-{ }^{15} \mathrm{~N} 22 \mathrm{kDa} \mathrm{GH}$ ) is spiked as the internal standard to both serum sample and calibrator at the beginning of the procedure. T6 and T12 given in red letters are to indicate the isotope-labeled versions of the fragments.

GH: Growth hormone; RP: Reversed-phase; SCX: Strong cation exchange. Reproduced with permission from [19] @ European Society of Endocrinology (2014).

For color images please see www.future-science.com/doi/full/10.4155/ bio.14.196.

for accurate recovery of $\mathrm{GH}$ and indicate the absence of interferences. Apart from a bilateral comparison [15], no comprehensive interlaboratory study has been run yet for MS-measurement of GH. However, results 
Table 1. Recovery of $22 \mathrm{kDa}$ growth hormone from serum by mass spectrometry.

\begin{tabular}{lll|}
\hline & \multicolumn{2}{c|}{ Recovery (\%) } \\
\cline { 2 - 3 } & By T6 & By T12 \\
\hline 4.63 & 103.5 & 104.1 \\
\hline 4.58 & 97.2 & 100.4 \\
\hline 10.19 & 100.5 & 102.9 \\
10.25 & 98.9 & 99.0 \\
\hline 19.51 & 103.9 & 102.5 \\
\hline 19.32 & 102.0 & 96.8 \\
\hline 29.39 & 104.0 & 99.3 \\
30.31 & 103.1 & 100.4 \\
\hline Mean recovery (\%) & 101.6 & 100.7 \\
\hline CV (\%) & 2.5 & 2.4 \\
\hline LoQ (ng/ml) & 1.7 & 2.7 \\
\hline $\begin{array}{l}\text { CV: Coefficient of variation; LoQ: Limit of quantification. } \\
\text { Adapted with permission from [13] @ Elsevier Inc. (2010). }\end{array}$ \\
\hline
\end{tabular}

obtained by Cox et al. [28] in a recent study about IGF-1, constituting a similar analytical challenge as $\mathrm{GH}$, are promising. IGF-1 is particularly interesting by its close physiological relationship with GH. In that study, five laboratories, using different LC/MS-MS platforms demonstrated comparability within 5.6\%. $\mathrm{U}-{ }^{15} \mathrm{~N}$ IGF-1 was employed as isotope labeled material in this example.

Selectivity and sensitivity achievable with MS is further illustrated by the example shown in Figure 5, with no other signals present in the ion chromatograms, but those at the known retention times of the peptides T6 and T12. In contrast to presently prevailing perception $[29,30]$, this example of a serum-GH measurement

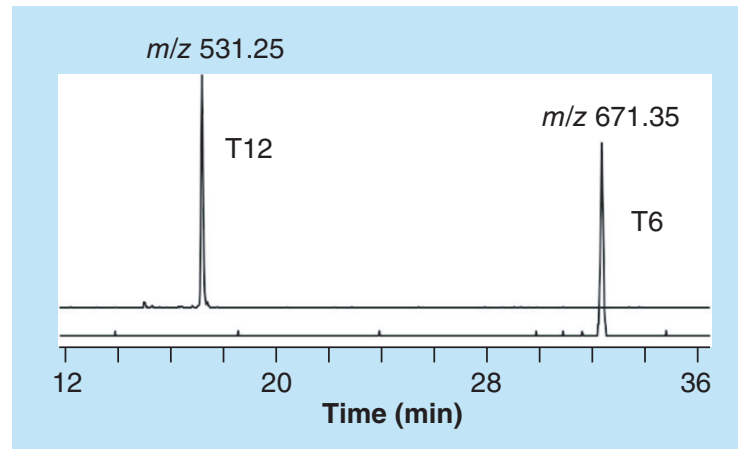

Figure 5. Signals obtained from a serum sample at $1 \mathrm{ng} / \mathrm{ml}$ growth hormone. Tryptic peptides T6 and T12 of growth hormone had been extracted and were then analyzed by LC/MS-MS using the masses of collision fragments at $m / z 531.25$ and 671.35 for monitoring. Instrument: Orbitrap-Elite at 60.000 mass resolution. Reproduced with permission from [19] () European Society of Endocrinology (2014). at $1 \mathrm{ng} / \mathrm{ml}$ also suggests that, MS-techniques can be run as sensitive, as making them competitive to contemporary antibody-based assays, even in concentration ranges relevant with glucose-tolerance tests, where cut-off levels have been reported of approximately $0.5 \mathrm{ng} / \mathrm{ml}$ [31].

Needless to elaborate that, selectivity and sensitivity are largely interrelated and many factors in sample preparation, as well as instrumental set-up, have to be optimized so as to touch the limits of what is technically possible. The first version [13] of the protocol outlined in Figure 4 was developed on a triple-quadrupole MS instrument. Monitoring collision fragments of T6 and T12, rather than unfragmented ions, essentially contributes to selectivity, as including a sequencedependent parameter (fragment mass) for further distinction from matrix components, that might chance to have the same mass as T6 (or T12). The result shown in Figure 5, however, was obtained under conditions of high mass resolution as achieved by using an Orbitrap mass spectrometer in place of the quadrupole instrument. This, apparently, does not just further increase selectivity, but it also improves the signal-to-noise ratio, and sensitivity, thus. Another option to enhance sensitivity consists in peptide conjugation to enhance amphipathicity, and ionization yield, by this way. So, for example, based on selective tagging at cysteine, the limit of quantification could be improved by a factor of 4 in a later published variant of the protocol [14].

\section{Susceptibility to interfering proteins}

Owing to the advantage of high selectivity, as pointed out, mass spectrometric quantification of $\mathrm{GH}$ is not likely to be biased in presence of whatever background of accompanying proteins. Consequently, interference by GHBP, as commonly observed with many LBAs, is expected to be irrelevant with MS-based measurement.

This is affirmed by the data shown in Figure 6. Samples from a serum pool with defined concentration of $\mathrm{GH}(9 \mathrm{ng} / \mathrm{ml})$ had been fortified with varying amounts of binding protein, such as reflecting the clinically relevant concentration range. The MS-method accurately recovers the total amount of $\mathrm{GH}$, while the antibodybased assays report only the fraction that is accessible to the antibodies at the equilibrium of competitive binding.

Obviously, MS is highly reliable if it is about the total amount of GH in the sample. At the same time, it does not, of course, resolve in what aggregation state (free, receptor-bound and dimerized, among others) the hormone is present. It cannot be ruled out, however, that these fractions contribute essential information to description of the physiological status of a patient and might evolve as relevant measurands too. 


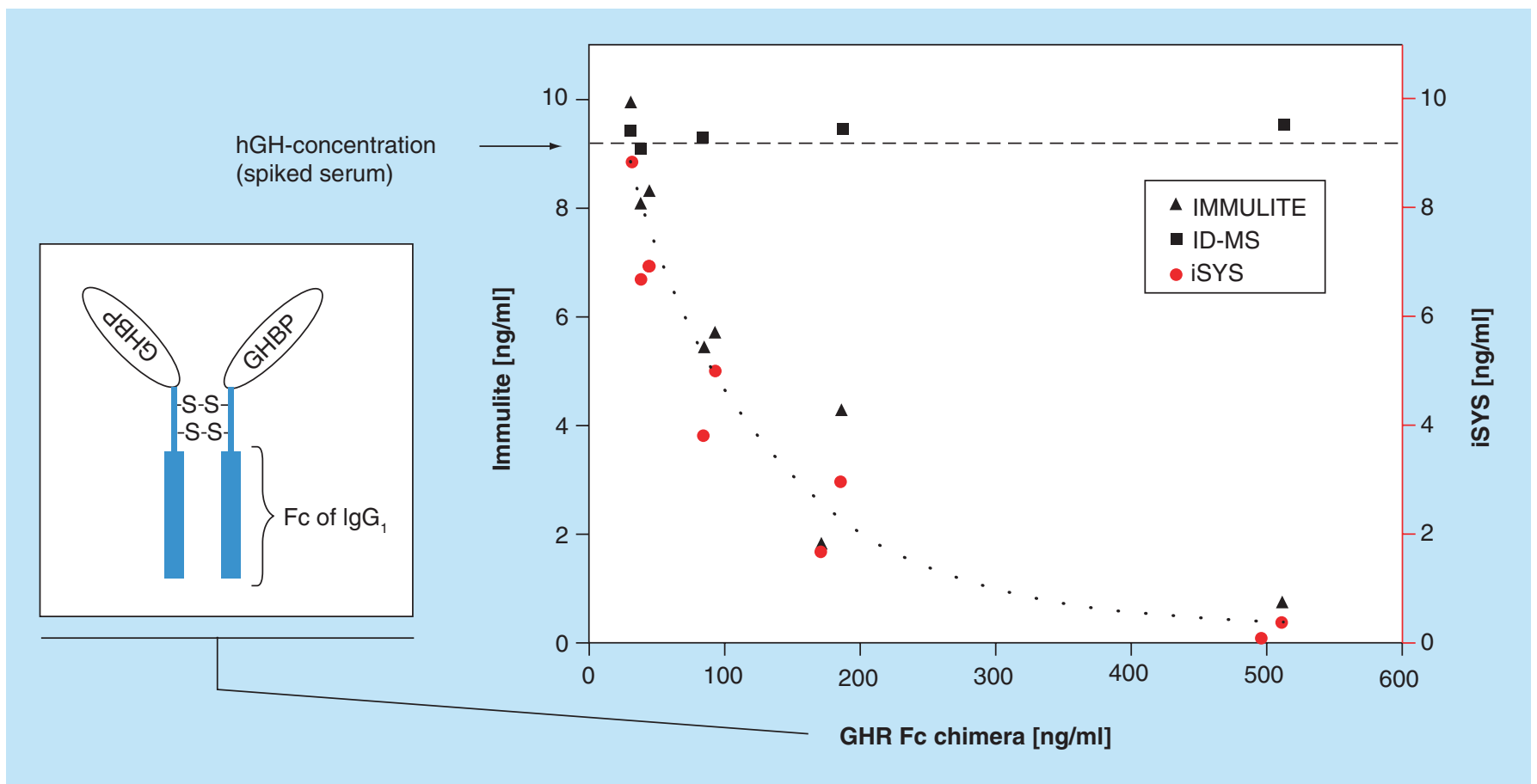

Figure 6. Dependence of serum growth hormone assay results on binding protein (GHBP) level. Two commonly used antibody-based assays (IMMULITE, triangles and iSYS, circles) are compared with mass spectrometry (ID-MS, squares). A GHR Fc chimera was spiked to serum so as to mimic increasing concentrations of the binding protein. The dashed horizontal line indicates the known concentration of $22 \mathrm{kDa}$ growth hormone. ID-MS data shown are averages from T6-based and T12-based GH measurement.

Reproduced from [32].

Taking account of this, Frystyk et al. [33] used ultrafiltration to remove the receptor-bound fraction of $\mathrm{GH}$ prior to quantification of the remaining 'free GH'. A correlation was found, by these authors, between total and free GH for a given (constant) level of GHBP. This suggests redundance between the two measurands. If so, just one of the two would be needed for diagnostic purposes in reality, provided the GHBP level be known at the same time for that sample. Unfortunately, no MS-method, has been reported yet for GHBP-quantification, but could be useful in the context.

Frystyk et al. also found an inverse correlation of free-GH and GHBP levels (for a given total amount of GH). This is supportive of the notion that, GHBP serves to control the levels of 'active' GH available to the receptors. The example illustrates, that there may be no single and simple measurand sufficiently describing the clinically relevant interaction of the different molecular players in $\mathrm{GH}$ signaling and metabolism.

Returning on the high-selectivity advantage by MS, it should be mentioned that, GH-measurement by MS might cope with presence of pegvisomant, even in high excess, in a similar way, as it does with GHBP. This, of course, requires signature peptides to be selected, which are different between GH and pegvisomant.

\section{Providing reference values}

MS-measurement, as pointed out, enables acquisition of SI-traceable values for (total) GH, which can serve either as reference values for this measurand with quality control, or for calibration of LBAs.

Viability of calibration of LBAs, concedingly, is limited by the fact that, the (unknown) GHBP level is involved in the outcome. Obviously, correct results can be only expected, if this level is the same in the calibrator as with the particular sample. This, however, is not the case, most of the time. Dissociation of GHBP-antigen complexes under acidic conditions, eliminating interference, thus, of the binding protein, has been proposed as a strategy for circumventing the problem [34].

Recently, Physikalisch-Technische Bundesanstalt has started to provide MS-values for intercomparisons as shown in Figure 2. The capability of SI-traceable [35] MS-based GH-measurement is supported on the international level by a 'Calibration and Measurement Capability (CMC)' registered at the Bureau International des Poids et Mesures (BIPM) [36].

\section{Key term}

BIPM: The task of the BIPM is to ensure world-wide uniformity of measurements and their traceability to the International System of Units (SI). 
Capturing isoform-specific

Up to this point, discussion of the MS method was restricted by the assumption that, the $22 \mathrm{kDa} \mathrm{GH}$ form exclusively appears in a sample. However, the concept of quantifying $\mathrm{GH}$ by signature peptides allows for separate measurement of the minor abundant GH forms too, which are present in a natural sample next to $22 \mathrm{kDa} \mathrm{GH}$.

T6, for instance, as it is shown in Figure 3, selectively captures the $22 \mathrm{kDa}$ GH form. The peptide cannot result from $20 \mathrm{kDa} \mathrm{GH}$, as the amino acids within the grey box would miss with this form (next to further deviations). For quantification of the $20 \mathrm{kDa}$ form, on the other hand, $(20 \mathrm{kDa}$ GH) T4 would be one out of the possible signature peptides. (T4 refers to the fourth tryptic peptide produced from the protein, starting enumeration from the $\mathrm{N}$-terminus).

In a similar way, numerous peptides can be specified, which selectively measure a particular form of $\mathrm{GH}$ that is of interest in a particular study. In most of the cases, more than just one cleavage product is available to represent the same GH form. If simultaneously quantifying more than one, the redundancy can be used to enhance reliability by averaging the results. Also important to observe, the individual results will additionally provide 'checks and balances'. In the case of discrepancy, particularly, if one fragment should not be detected at all, this may indicate an unexpected structural deviation in the GH sequence with that particular sample, altering the molecular mass of that peptide. Changes like this might result from, for example, mutation (replacement of amino acid) or side-chain modification.

\section{Revising measurands}

The option of separately monitoring different GH forms in high-selectivity/high-reliability mode will foreseeably impact progress in GH research too. Based on MS-measurement, decisions could become possible, where data from LBAs are leaving things ambiguous, presently.

One example, where such progress might be anticipated, is about the influence of the spectrum of circulating $\mathrm{GH}$-isoforms on the occurrence of short stature in children. Different studies lead to different conclusions: While results by Boguszewski et al. [37] suggested that elevated levels of non- $22 \mathrm{kDa}$ forms (which mainly is $20 \mathrm{kDa} \mathrm{GH}$ ) are responsible for the (patho-)physiological condition, Tsushima et al. [38] reported constant

\section{Key term}

Isoform-differential test: Test based on change in abundance ratio(s) of isoforms. ratios of $20 \mathrm{kDa}$ and $22 \mathrm{kDa} \mathrm{GH}$, regardless of healthy or short stature. Also, it was proposed [39] that, minor isoforms (neither $22 \mathrm{kDa}$, nor $20 \mathrm{kDa}$ ) might account for the effects observed by Boguszewski et al.

An MS-based study may be expected to reduce ambiguity by providing better analytical resolution, as resulting from mass-selective differentiation of isoforms.

\section{Defining cut-off levels}

The determination of diagnostic decision levels generally involves lengthy and elaborate clinical studies. It is particularly important therefore, to have the measurand well-defined at the outset. As it has been pointed out, present knowledge about what is the optimal measurand in GH-related diseases (sum of GH forms, individual isoform[s], bound/free ratio, other?) may be supported by MS-methods of measurement.

A major limitation associated with exclusive reference to LBA measurement in determination of cut-off values is that, the antibody may not be available anymore at a later point, as well as the calibrator be replaced by another one, which changes will hamper applicability (reproducibility) then, of results.

MS, as opposed to this, has the potential of establishing sustainable reproducibility, independent of what laboratory or MS-platform being used. An example for MS-based cut-off determination in diagnosis of GH deficiency is given in Figure 7. In spite of the limited size of the data set ( $\mathrm{n}=30$ patients), it turns out that, both, T6 and T12, appear to equally discriminate between healthy and GH-deficient children. The ratio between the cut-offs obtained for T6 and T12, 5.9 and $6.8 \mathrm{ng} / \mathrm{ml}$, is roughly reflecting the expected fraction of $22 \mathrm{kDa}$ GH within total GH (here: $87 \%$ ). In the original study [19], MS was employed to establish a metrological link to the LBA-based cut-off values.

\section{Supporting doping control}

Present detection strategies for GH-doping in sports have been reviewed by Baumann et al. [40]. The particular potential of MS-based proteomics methods for detection of protein doping has been highlighted by Kay et al., [29] and a review, summarizing recent development in the area, has been published by van den Broek et al. [30]. Insufficient sensitivity uniformly was discussed to be the main obstacle to application of MS. However, the examples and data provided in the previous sections indicate that, the sensitivity needed may be attainable if applying the latest generation of MSinstruments in combination with optimized sample preparation.

In $\mathrm{GH}$ doping, recombinant $22 \mathrm{kDa} \mathrm{GH}$, putatively, is the common form of administration. The intake will increase the serum GH-level for about $20 \mathrm{~h}$ 
(window of opportunity in doping control). Nevertheless, definition of an (absolute) cut-off concentration, as a way to unambiguously detect such event, is not viable: fluctuations from as little as $0.01 \mathrm{ng} / \mathrm{ml}$ up to approximately $20-30 \mathrm{ng} / \mathrm{ml}$ (or even more) are not uncommon, as resulting from just normal biorhythm, with a peak-to-peak interval of 2-3 h. In addition, elevated $\mathrm{GH}$ levels may also occur as response to physical exercise, stress or fasting [40].

Therefore, 'isoform-differential' tests have been developed which do not depend on absolute concentrations of the hormone. Instead, the disturbance of the natural spectrum of isoforms, which results from exogenous administration of the $22 \mathrm{kDa} \mathrm{GH}$, is used as an indicator. Based on this notion, Wu et al. [41] and Bidlingmaier et al. [42], for example, developed methods using a combination of antibodies recognizing different GH-fractions, each: one type of antibodies specifically measures only $22 \mathrm{kDa}$ GH ('rec GH'), while the other one recognizes all forms secreted by the pituitary ('pit GH'). Elevated rec/pit-ratios are taken indicative of doping with $22 \mathrm{kDa}$ GH. Momomura et al. [43], in contrast to this, proposed a combination of antibodies, which both are isoform-specific, targeting the $22 \mathrm{kDa}$ and the $20 \mathrm{kDa}$-form, respectively. The principle is illustrated in Figure 8.

Exploration of the potential of MS in this area is suggesting itself. MS-based acquisition of isoform ratios could be expected to either directly improve discrimination between both classes of samples (doped or not), or, at least, be used to back-up findings by LBAs. The epitope-recognition principles of the existing tests can be translated into signature peptides for MS-measurement in a straightforward way: T6/T12-ratios, for example, may be deemed to map a quantity corresponding to the rec/pit assay, as T6 selectively measures $22 \mathrm{kDa}$ GH, while T12 represents 'total GH' (Figure 3). Replacing T12 by T4 (confined to the substretch exclusively present on $20 \mathrm{kDa} \mathrm{GH}$ ), measuring T6/T4-ratios thus, would constitute an MS-analogy to the method of Momomura et al. [43].

\section{Conclusion \& future perspective}

The previous sections support the conclusion that, MS provides an effective alternative to traditional (antibody-based) LBAs in GH-measurement. Advantages of MS result from: high analytical selectivity enabled by mass-recognition instead of epitopes; flexibility and precision in targeting particular (iso-)forms by using signature peptides; and ease of integration of isotope labeled internal standards into the protocol. By virtue of these features, many of the problems encountered with LBAs either do not occur at all with MS-methods, or may be overcome in a simple way. This does not just

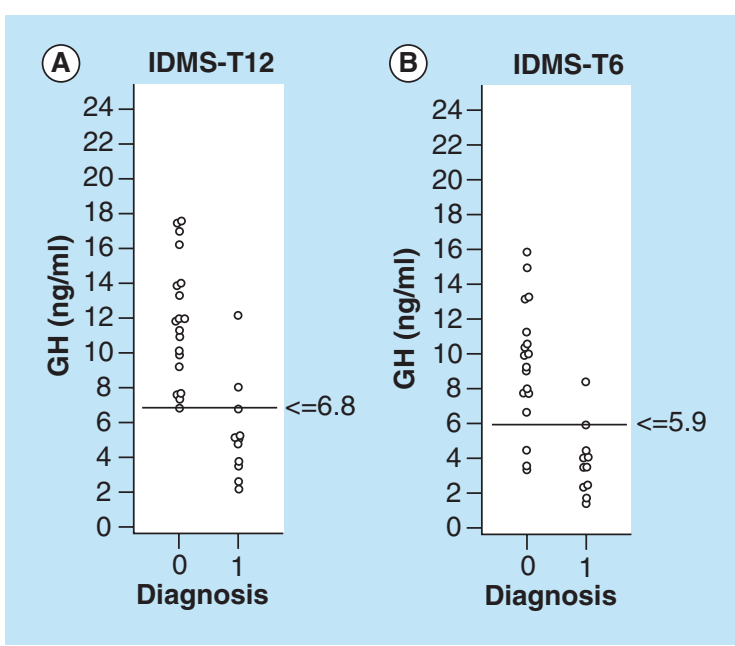

Figure 7. MS in definition of diagnostic cut-off levels. Results are shown for healthy (0) and GH-deficient children (1). Signature peptides (A) T12 and (B) T6 were quantified. Monitoring T12 is equivalent to mapping 'total-GH' whereas T6 reflects $22 \mathrm{kDa} \mathrm{GH}$.

$\mathrm{GH}$ : Growth hormone.

Graphical representation of data contributed to a recent publication [19].

apply to interferences with binding- or other proteins, and commutability issues resulting therefrom, but also to imprecisions in definition/recognition of the targeted epitope or GH form, in a more general sense.

The technology has matured to the extent as being available for either direct measurement of patient samples (thus replacing LBAs), or value-assignment to serum-based control- and reference materials (supporting calibration and proficiency testing of LBAs).

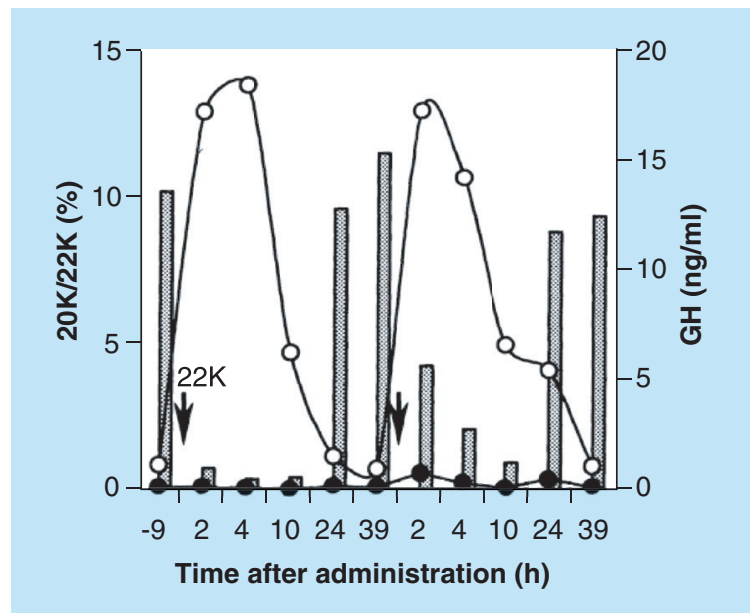

Figure 8. Effect of doping on isoform composition. Changes in serum concentrations of $20 \mathrm{kDa}$ GH (black circles) and $22 \mathrm{kDa}-\mathrm{GH}$ (white circles) and the ratio of $20 \mathrm{kDa} / 22 \mathrm{kDa}$ (bar) after administration of $22 \mathrm{kDa} \mathrm{GH}$. The arrows represent the time of administration. $\mathrm{GH}$ : Growth hormone.

Reproduced with permission from [43] () The Japan Endocrine Society (2000). 
Standardization efforts for present commercial tests, no doubt, can capitalize on comparison with MS-results. Equally important it is, however, that the option of highly selective (iso-)form-specific GH-quantification by MS has much promise as analytical tool to support further investigation into the putatively different biological functions of these forms, and if different, which particular functions these are.

Future development should aim at further improving sensitivity in measurement of less abundant isoforms (e.g., T4-based monitoring of $20 \mathrm{kDa} \mathrm{GH}$, and similar). Likewise, validation of method applicability in practice should be demonstrated by MS-based interlaboratory studies. Rationalization in terms of turnaround time, as much as possible, will influence acceptance of the method principle in practice, of course, too.

However, the advantages associated with MS-based measurement eventually might outweigh the main assets of LBAs, as low costs and ease of use in routine. The more so, if considering that, results are scarcely needed within a few hours in diagnosis of GH-related disorders. The time, MS analysis takes, and higher costs per analysis, in practice might pay off in terms of more accurate classification of patients, and of costs avoided, which would result from inappropriate treatment.

Financial \& competing interests disclosure

The authors have no relevant affiliations or financial involvement with any organization or entity with a financial interest in or financial conflict with the subject matter or materials discussed in the manuscript. This includes employment, consultancies, honoraria, stock ownership or options, expert testimony, grants or patents received or pending, or royalties.

No writing assistance was utilized in the production of this manuscript.

\section{Open access}

This work is licensed under the Creative Commons Attribution-NonCommercial 3.0 Unported License. To view a copy of this license, visit http://creativecommons.org/licenses/by$n c-n d / 3.0$

Executive summary

Measurand

- Growth hormone (GH) as circulating in blood consists of many chemically slightly different (iso-)forms, which is posing the challenge of accurate and specific measurement.

Recognizing by epitope

- Performance of antibody-based ligand-binding assays in isoform-specific detection of GH suffers from limited analytical resolution as inherent to the principle of analyte recognition by epitope.

Recognizing by mass

- Mass spectrometry (MS), by high mass resolution attainable, can provide the selectivity needed. In addition, integration of isotope labeled GH into the workflow imparts high reliability to quantitative MS results.

GH by MS: scope \& bounds

- Most promising applications of MS-based GH measurement are: direct measurement of patient samples; acquisition of reference values for calibration of routine ligand-binding assays; and isoform-specific measurement in support of research into particular biological functions of these forms.

\section{References}

Papers of special note have been highlighted as: - of interest

1 Baumann GP. Growth hormone isoforms. Growth Horm. IGF Res. 19, 333-340 (2009).

- The abuse of growth hormone $(\mathrm{GH})$ in sport and its detection: a medical, legal and social framework. Comprehensive up-to-date compendium about GH heterogeneity.

2 Sinha YN, Jacobsen BP. Human growth hormone (hGH)-(44-191), a reportedly diabetogenic fragment of hGH, circulates in human blood: measurement by radioimmunoassay. J. Clin. Endocrinol. Metab. 78, 1411-1418 (1994).
3 Bidlingmaier M, Freda PU. Measurement of human growth hormone by immunoassays: current status, unsolved problems and clinical consequences. Growth Horm. IGF Res. 20, 19-25 (2010).

4 Müller A, Scholz M, Blankenstein O et al. Harmonization of growth hormone measurements with different immunoassays by data adjustment. Clin. Chem. Lab. Med. 1135-1142 (2011).

5 Wieringa GE, Sturgeon CM, Trainer PJ. The harmonisation of growth hormone measurements: taking the next steps. Clin. Chim. Acta. 432, 68-71 (2014).

- Provides an inventory about the actual state of interlaboratory comparability of GH results, and summarizes harmonization efforts toward further improvement. 
6 Arafat AM, Möhlig M, Weickert MO et al. Growth hormone response during oral glucose tolerance test: the impact of assay method on the estimation of reference values in patients with acromegaly and in healthy controls, and the role of gender, age, and body mass index. J. Clin. Endocrinol. Metab. 93, 1254-1262 (2008).

7 Paisley AN, Hayden K, Ellis A, Anderson J, Wieringa G, Trainer PJ. Pegvisomant interference in GH assays results in underestimation of GH levels. Eur. J. Endocrinol. 156, 315-319 (2007).

8 Boulo S, Hanisch K, Bidlingmaier M et al. Gaps in the traceability chain of human growth hormone measurements. Clin. Chem. 59, 1074-1082 (2013).

9 Bristow AF, Jespersen AM. The second international standard for somatropin (recombinant DNA-derived human growth hormone): preparation and calibration in an international collaborative study. Biologicals 29, 97-106 (2001).

- Marks the introduction of $22 \mathrm{kDa} \mathrm{GH}$ as basis for calibration of ligand-binding assay (LBA), establishing a link, thus, to (molar) amount-of-substance as SI-unit.

10 Miller WG, Myers GL, Rej R. Why commutability matters. Clin. Chem. 52, 553-554 (2006).

11 Le Breton M-H, Rochereau-Roulet S, Pinel G et al. Direct determination of recombinant bovine somatotropin in plasma from a treated goat by liquid chromatography/ high-resolution mass spectrometry. Rapid Commun. Mass Spectrom. 22, 3130-3136 (2008).

12 Bailly-Chouriberry L, Pinel G, Garcia P, Popot M-A, Le Bizec B, Bonnaire Y. Identification of recombinant equine growth hormone in horse plasma by LC-MS/MS: a confirmatory analysis in doping control. Anal. Chem. 80, 8340-8347 (2008).

13 Arsene CG, Henrion A, Diekmann N, Manolopoulou J, Bidlingmaier M. Quantification of growth hormone in serum by isotope dilution mass spectrometry. Anal. Biochem. 401, 228-235 (2010).

14 Arsene CG, Schulze D, Kratzsch J, Henrion A. High sensitivity mass spectrometric quantification of serum growth hormone by amphiphilic peptide conjugation. J. Mass Spectrom. 47, 1554-1560 (2012).

15 Pritchard C, Groves KJ, Biesenbruch S et al. The quantification of human growth hormone in serum with a labeled protein as an internal standard: essential considerations. Anal. Chem. 86(13), 6525-6532 (2014).

16 Geng M, Ji J, Regnier FE. Signature-peptide approach to detecting proteins in complex mixtures. J. Chromatogr. A 870, 295-313 (2000).

17 Ruan Q, Ji QC, Arnold ME, Humphreys WG, Zhu M. Strategy and its implications of protein bioanalysis utilizing high-resolution mass spectrometric detection of intact protein. Anal. Chem. 83, 8937-8944 (2011).

18 Gucinski AC, Boyne MT. Evaluation of intact mass spectrometry for the quantitative analysis of protein therapeutics. Anal. Chem. 84, 8045-8051 (2012).

19 Wagner I, Pätzold C, Gausche R et al. Clinical evidence based decision limits for growth hormone stimulation tests in children with back-up of results by reference to mass spectrometry. Eur. J. Endocrinol. 171(3), 389-397 (2014).

20 Brun V, Dupuis A, Adrait A et al. Isotope-labeled protein standards: toward absolute quantitative proteomics. Mol. Cell Proteomics 6, 2139-2149 (2007).

21 Lebert D, Dupuis A, Garin J, Bruley C, Brun V. In gel-free proteomics. In: Methods in Molecular Biology (Volume 753). Gevaert K, Vandekerckhove J (Eds), Humana Press, NY, USA, 93-115 (2011).

22 Arsene CG, Ohlendorf R, Burkitt W et al. Protein quantification by isotope dilution mass spectrometry of proteolytic fragments: cleavage rate and accuracy. Anal. Chem. 80, 4154-4160 (2008).

23 Ackermann BL, Berna MJ. Coupling immunoaffinity techniques with MS for quantitative analysis of low-abundance protein biomarkers. Expert Rev. Proteomics 4, 175-186 (2007).

24 Weiss F, van den Berg BH, Planatscher H, Pynn CJ, Joos TO, Poetz O. Catch and measure - mass spectrometry-based immunoassays in biomarker research. Biochim. Biophys. Acta 1844(5), 927-932 (2014).

25 Anderson NL, Anderson NG, Haines LR, Hardie DB, Olafson RW, Pearson TW. Mass spectrometric quantitation of peptides and proteins using stable isotope standards and capture by anti-peptide antibodies (SISCAPA). J. Proteome Res. 3, 235-244 (2004).

26 Becker JO, Hoofnagle AN. Replacing immunoassays with tryptic digestion-peptide immunoaffinity enrichment and LC-MS/MS. Bioanalysis 4, 281-290 (2012).

27 Burkitt WI, Pritchard C, Arsene C, Henrion A, Bunk D, O'Connor G. Toward Système International d'Unité - traceable protein quantification: from amino acids to proteins. Anal. Biochem. 376, 242-251 (2008).

28 Cox, HD, Lopes, F, Woldemariam, GA et al. Interlaboratory agreement of insulin-like growth factor 1 concentrations measured by mass spectrometry. Clin. Chem. 60, 541-548 (2014).

29 Kay RG, Creaser CS. Application of mass spectrometry-based proteomics techniques for the detection of protein doping in sports. Expert Rev. Proteomics 7, 185-188 (2010).

30 van den Broek I, Blokland M, Nessen MA, Sterk S. Current trends in mass spectrometry of peptides and proteins: Application to veterinary and sports-doping control. Mass Spectrom. Rev. doi:10.1002/mas.21419 (2013) (Epub ahead of print).

- Reviewing latest state of the art in MS technologies for detection of illicit administration/intake of polypeptides in sport doping, as well as stock-breeding.

31 Bancos I, Algeciras-Schimnich A, Woodmansee WW et al. Determination of nadir growth hormone concentration cutoff in patients with acromegaly. Endocr. Pract. 19, 937-945 (2013).

32 Arsene CG, Kratzsch J, Henrion A. Interference of binding protein with serum growth hormone measurements. ArXiv 1405.3895 (2014).

33 Frystyk J, Andreasen CM, Fisker S. Determination of free growth hormone. J. Clin. Endocrinol. Metab. 93, 3008-3014 (2008). 
34 Myler HA, McVay S, Kratzsch J. Troubleshooting PEG-hGH detection supporting pharmacokinetic evaluation in growth hormone deficient patients. J. Pharmacol. Toxicol. Methods 61(2), 92-97 (2010).

35 BIPM - VIM.

www.bipm.org/en/publications/guides/vim.html

36 BIPM - home page.

www.bipm.org/en/home

37 Boguszewski CL, Jansson C, Boguszewski MCS et al. Increased proportion of circulating non-22-kilodalton growth hormone isoforms in short children: a possible mechanism for growth failure. J. Clin. Endocrinol. Metab. 82, 2944-2949 (1997).

38 Tsushima T, Katoh Y, Miyachi Y et al. Serum concentration of $20 \mathrm{~K}$ human growth hormone (20K hGH) measured by a specific enzyme-linked immunosorbent assay. J. Clin. Endocrin. Metab. 84, 317-322 (1999).

39 Wallace JD, Cuneo RC, Bidlingmaier M et al. The response of molecular isoforms of growth hormone to acute exercise in trained adult males. J. Clin. Endocrinol. Metab. 86, 200-206 (2001).
40 Baumann GP. Growth hormone doping in sports: a critical review of use and detection strategies. Endocr. Rev. 33, 155-186 (2012).

41 Wu Z, Bidlingmaier M, Dall R, Strasburger CJ. Detection of doping with human growth hormone. Lancet 353, 895 (1999).

- First time that isoform-differential measurement and use of abundance ratios has been proposed for detection of GH-doping.

42 Bidlingmaier M, Suhr J, Ernst A et al. High-sensitivity chemiluminescence immunoassays for detection of growth hormone doping in sports. Clin. Chem. 55, 445-453 (2009).

43 Momomura S, Hashimote Y, Shimazaki Y, Irie M. Detection of exogenous growth hormone $(\mathrm{GH})$ administration by monitoring ratio of $20 \mathrm{kDa}$ - and $22 \mathrm{kDa}-\mathrm{GH}$ in serum and urine. Endocr. J. 47, 97-101 (2000).

44 RfB - Referenzinstitut für Bioanalytik. www.dgkl-rfb.de 